\title{
The Clinical and Research Implications of Anti-carbamylated Protein Antibodies
}

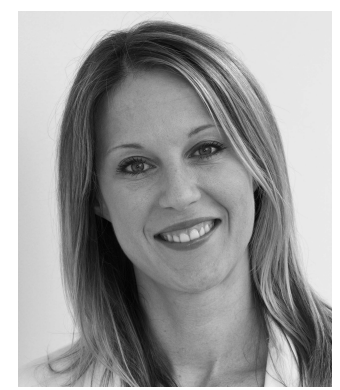

Autoantibodies play a central role in the clinical management of rheumatoid arthritis (RA). They can be used for diagnostic as well as prognostic purposes. For many years, autoantibody testing in RA was limited to rheumatoid factor (RF), but in the late 1990s, Schellekens and colleagues confirmed that autoantibodies reactive to citrullinated peptides were highly specific for RA ${ }^{1}$. The identification of antibodies to citrullinated peptide/protein antigens (ACPA) and the commercialization of ACPA testing in the form of anticyclic citrullinated peptide (CCP) antibody assays has revolutionized the field of rheumatology. The magnitude of the role of autoantibodies in RA is reflected in the current 2010 RA classification criteria in which autoantibodies can account for up to 3 of the 6 points $(50 \%)$ needed to classify inflammatory arthritis as $\mathrm{RA}^{2}$.

In the clinical management of patients with RA, earlier diagnosis and initiation of appropriate treatment is associated with improved longterm outcomes ${ }^{3}$. While there are many factors that influence delays in diagnosis and treatment of RA, autoantibodies are one factor that can influence these clinical outcomes. For example, Pratt and colleagues found that in patients referred to an early arthritis clinic who were ultimately diagnosed with RA, patients who were ACPA- and RF-negative had a significant delay in the time to treatment following assessment by a rheumatologist ${ }^{4}$. This finding highlights the diagnostic uncertainty that rheumatologists often face when patients with inflammatory arthritis are negative for disease-specific autoantibodies. Further, it highlights the importance of identifying novel autoantibody systems in RA, particularly ones that can help to identify the $20 \%-30 \%$ of patients with RA who are seronegative for RF and ACPA.

Notably, it remains unknown whether RA patients without detectable autoantibodies are truly seronegative or whether they possess an autoantibody that has yet to be discovered. Multiple investigations have sought to identify novel autoantibody biomarkers in RA. One particular autoantibody system that has received considerable attention in recent years has been anti-carbamylated protein (anti-CarP) antibodies in RA. Carbamylation is a post-translational chemical modification induced by cyanate that results in peptidyl-homocitrulline. Like citrullination, carbamylation can occur during common physiologic processes such as inflammation, and specifically as part of a respiratory burst that may occur in the setting of pathogens encountered at a mucosal surface ${ }^{5}$; however, the generation of an antibody response to CarP is uncommon in healthy controls but present in a portion of patients with RA. This finding was first reported in the study by Shi and colleagues in which they demonstrated that autoantibodies to CarP were elevated in $45 \%$ of patients with RA and were highly specific for RA compared to controls ${ }^{6}$. This study also found that a portion of ACPA-negative patients with RA had anti-CarP antibodies, and while the RF status was not reported for patients with RA in this study, this finding suggested that anti-CarP antibodies may be useful to reduce the diagnostic uncertainty in a portion of seronegative patients with inflammatory arthritis.

To effectively use anti-CarP antibodies in the clinical diagnosis of RA, particularly ACPA/RF-negative RA, it is necessary to understand the performance of anti-CarP antibodies in non-RA connective tissue diseases (CTD) because other CTD are often on the differential in a patient presenting with inflammatory arthritis. In this issue of The Journal, Nakabo and colleagues ${ }^{7}$ report the prevalence of anti-CarP antibodies in patients with RA as well as patients with a variety of other CTD. This study found that $47 \%$ of patients with RA, including $21 \%$ of ACPA-negative patients with RA, had anti-CarP antibody positivity. Compared to healthy controls, anti-CarP was highly specific for RA (97\%). However, the authors also found that 146/620 (24\%) of non-RA patients with CTD demonstrated anti-CarP positivity, making the specificity of anti-CarP only $76 \%$ when compared to patients with non-RA CTD. The highest prevalence of positivity for anti-CarP in non-RA CTD was demonstrated in patients with mixed CTD and overlap syndrome $(38 \%)$, primary Sjögren syndrome (36\%),

See Anti-CarP antibodies in CTD, page 1384

Personal non-commercial use only. The Journal of Rheumatology Copyright @ 2017 . All rights reserved. 
systemic sclerosis (23\%), systemic lupus erythematosus (SLE; 22\%), and vasculitis (22\%). Anti-CarP was also present in $50 \%$ of patients with spondyloarthritis, although only 8 patients were studied in this group. These results build on other recent studies including one by Bergum and colleagues that similarly found an increased prevalence of anti-CarP antibodies in patients with primary Sjögren syndrome ${ }^{8}$.

With these results, the authors conclude that anti-CarP antibodies alone cannot differentiate ACPA-negative RA from non-RA CTD. This is an interesting and important finding to appropriately interpret these antibody results clinically. However, in this study, little is known about concomitant arthritis in the non-RA patients with CTD. The authors report that there was no significant correlation between anti-CarP antibodies and arthritis in the subset of patients with SLE studied; however, the details of arthritis in these patients (e.g., joint distribution, arthritis severity, or methodology used to detect arthritis) are not reported. In addition, it is unknown whether the ACPA-negative patients with RA are also RF-negative, and this distinction is necessary to fully understand the clinical effect of anti-CarP testing. It may be that a more clinically useful question would be whether anti-CarP antibodies can distinguish ACPA/RF-negative RA from non-RA CTD with peripheral inflammatory arthritis, but this distinction cannot be made from the current study.

Additional limitations of the study that should be considered are that the diagnosis of each CTD was based on a physician's judgment rather than disease-specific classification criteria, anti-CarP antibody detection was performed on an in-house assay, and limited demographic information was provided to characterize the healthy control group that was used to establish the cutoff level for anti-CarP positivity. That being said, the study did confirm successful carbamylation of the assay antigen by mass spectrometry, and the rates of anti-CarP positivity in patients with RA were in line with rates reported in other studies. Importantly, the authors did not identify a significant correlation between anti-CarP and anti-CCP antibody positivity, suggesting that the antiCarP antibodies identified in these subjects were not simply cross-reactive anti-CCP antibodies.

While this study has important clinical implications as discussed above, these results may also have broader research implications regarding our understanding of disease pathogenesis for RA as well as other CTD. Studies support that ACPA can have a direct effect on joint disease pathogenesis ${ }^{9}$, and based on prior studies that found anti-CarP was associated with more severe joint damage in RA, it may be that anti-CarP antibodies also play a pathogenic role ${ }^{6}$. In addition, it has been suggested that RA-related autoantibodies may originate at a mucosal site such as the lung ${ }^{10}$. There was also a recent study by Skopelja and colleagues that demonstrated anti-CarP antibodies in $40 \%$ of patients with cystic fibrosis, a chronic inflammatory lung disease ${ }^{11}$. Additional research is needed to understand whether anti-CarP antibodies play a role in mucosal autoimmunity in the lung in RA. Finally, the presence of anti-CarP antibodies in other CTD may suggest shared pathways of autoimmune disease development, and additional studies should pursue this possibility because it could ultimately lead to novel treatment targets that may be applicable to multiple CTD.

\section{KRISTEN DEMORUELLE, MD, University of Colorado Denver, Division of Rheumatology, Aurora, Colorado, USA.}

Address correspondence to Dr. M.K. Demoruelle, University of Colorado School of Medicine, Division of Rheumatology, 1775 Aurora Court, Mail Stop B-115, Aurora, Colorado 80045, USA.

E-mail: Kristen.Demoruelle@UCDenver.edu

\section{REFERENCES}

1. Schellekens GA, de Jong BA, van den Hoogen FH, van de Putte LB, van Venrooij WJ. Citrulline is an essential constituent of antigenic determinants recognized by rheumatoid arthritis-specific autoantibodies. J Clin Invest 1998;101:273-81.

2. Aletaha D, Neogi T, Silman AJ, Funovits J, Felson DT, Bingham $\mathrm{CO}$, 3rd, et al. 2010 Rheumatoid arthritis classification criteria: an American College of Rheumatology/European League Against Rheumatism collaborative initiative. Arthritis Rheum 2010; 62:2569-81.

3. Finckh A, Liang MH, van Herckenrode CM, de Pablo P. Long-term impact of early treatment on radiographic progression in rheumatoid arthritis: a meta-analysis. Arthritis Rheum 2006;55:864-72.

4. Pratt AG, Lendrem D, Hargreaves B, Aslam O, Galloway JB, Isaacs JD. Components of treatment delay in rheumatoid arthritis differ according to autoantibody status: validation of a single-centre observation using national audit data. Rheumatology 2016; 55:1843-8.

5. Wang Z, Nicholls SJ, Rodriguez ER, Kummu O, Horkko S, Barnard $\mathrm{J}$, et al. Protein carbamylation links inflammation, smoking, uremia and atherogenesis. Nat Med 2007;13:1176-84.

6. Shi J, Knevel R, Suwannalai P, van der Linden MP, Janssen GM, van Veelen PA, et al. Autoantibodies recognizing carbamylated proteins are present in sera of patients with rheumatoid arthritis and predict joint damage. Proc Natl Acad Sci U S A 2011;108:17372-7.

7. Nakabo S, Yoshifuji H, Hashimoto M, Imura Y, Nakashima R Murakami K, et al. Anti-carbamylated protein antibodies are detectable in various connective tissue diseases. J Rheumatol 2017;44:1384-8.

8. Bergum B, Koro C, Delaleu N, Solheim M, Hellvard A, Binder V, et al. Antibodies against carbamylated proteins are present in primary Sjogren's syndrome and are associated with disease severity. Ann Rheum Dis 2016;75:1494-500.

9. Harre U, Georgess D, Bang H, Bozec A, Axmann R, Ossipova E, et al. Induction of osteoclastogenesis and bone loss by human autoantibodies against citrullinated vimentin. J Clin Invest 2012;122:1791-802.

10. Demoruelle MK, Harrall KK, Ho L, Purmalek MM, Seto NL, Rothfuss HM, et al. Anti-citrullinated protein antibodies are associated with neutrophil extracellular traps in the sputum in relatives of rheumatoid arthritis patients. Arthritis Rheumatol 2017;69:1165-75

11. Skopelja S, Hamilton BJ, Jones JD, Yang ML, Mamula M, Ashare A, et al. The role for neutrophil extracellular traps in cystic fibrosis autoimmunity. JCI Insight 2016;1:e88912.

J Rheumatol 2017;44:1302-3; doi:10.3899/jrheum.170697

Personal non-commercial use only. The Journal of Rheumatology Copyright (C) 2017. All rights reserved. 